\title{
Unsteady flow and heat transfer over a permeable stretching/shrinking sheet with generalized slip velocity
}

\begin{abstract}
Purpose: This study aims to investigate the unsteady two-dimensional viscous flow and heat transfer over an unsteady permeable stretching/shrinking sheet (surface) with generalized slip velocity condition. Design/methodology/approach: Similarity transformation is used to reduce the system of partial differential equations into a system of nonlinear ordinary differential equations. The resulting equations are then solved numerically using "bvp4c" function in MATLAB software. Findings: Dual solutions are found for a certain range of the unsteady, suction and stretching/shrinking parameters. Stability analysis is performed, and it is revealed that the first (upper branch) solution is stable and physically realizable, whereas the second (lower branch) solution is unstable. Practical implications: The results obtained can be used to explain the characteristics and applications of the generalized slip in boundary layer flow. Such condition is applied for particulate fluids such as foams, emulsions, polymer solutions and suspensions. Furthermore, the phenomenon of stretching/shrinking sheet can be found on the manufacturing of polymer sheets, rising and shrinking balloon or moving and shrinking polymer film. Originality/value: The present numerical results are original and new for the study of unsteady flow and heat transfer over a permeable stretching/shrinking sheet with generalized slip velocity.
\end{abstract}

Keyword: Stability analysis; Dual solutions; Heat transfer; Unsteady flow; Stretching/shrinking sheet; Generalized slip 